\title{
Gaining insight from large data volumes with ease
}

\author{
Valentin Kuznetsov ${ }^{1, *}$ \\ ${ }^{1}$ Cornell University, Ithaca, NY, USA 14850
}

\begin{abstract}
.
Efficient handling of large data-volumes becomes a necessity in today's world. It is driven by the desire to get more insight from the data and to gain a better understanding of user trends which can be transformed into economic incentives (profits, cost-reduction, various optimization of data workflows, and pipelines). In this paper, we discuss how modern technologies are transforming well established patterns in HEP communities. The new data insight can be achieved by embracing Big Data tools for a variety of use cases, from analytics and monitoring to training Machine Learning models on a terabyte scale. We provide concrete examples within the context of the CMS experiment where Big Data tools are already playing or would play a significant role in daily operations.
\end{abstract}

\section{Introduction}

With the CERN LHC program underway, we start seeing an exponential growth of data volume in the High-Energy Physics (HEP) field ${ }^{1}$. In LHC Run 2, CERN experiments stored data volumes in the petabyte (PB) regime. For instance, in 2017 the CMS experiment alone produced around 30 billion raw events and complemented them with 16 billion Monte Carlo similated events. It successfully transferred a few PB/week with average transfer rates of 2-6 $\mathrm{GB} / \mathrm{s}$; almost 20PB of data were replicated at GRID T1 sites and about $80 \mathrm{~PB}$ at T2 sites. The disk utilization was at the level of 20/40/50PB at T0/T1/T2 sites respectively. With the upcoming High Luminosity LHC (HL-LHC) program at CERN all HEP experiments will face a new challenge, the exabyte $\left(10^{18}\right)$ era of computing [1]. We anticipate that new techniques and technologies will be required to handle this unprecedented amount of data. For example, the overall time of a typical physics analysis may be significantly reduced by moving away from sequential processing of events at GRID data centers to data-reduction facilities based on Big Data technologies [2]. Similarly, the experiment's metadata and services are undergoing significant changes by embracing data-processing on Hadoop+Spark platforms, and adding NoSQL databases with their traditional RDBMS counterparts to a growing list of data services.

In this paper, we discuss new technologies and techniques for handling experiment metadata to gain additional insight from distributed data sources, located at data centers, on HDFS, in relational and NoSQL databases. The new information obtained with parallel processing

\footnotetext{
*e-mail: vkuznet@gmail.com

${ }^{1}$ Here we refer to the data as raw and Monte Carlo (MC) data produced and processed by experiments as well as the associated metadata and distinguish them explicitly in the paper.
} 
of large datasets helps us better understand our resources, more efficiently utilize computing infrastructure, and gradually move towards a data-driven approach in the HL-LHC era of computing.

\section{Current landscape}

When the concept of relational databases was introduced in 1970 [3] it solved many problems in the world of information technology. In the HEP community the RDBMS technologies were used in many data services from online calibration to offline data bookkeeping systems as well as surrounding infrastructure. Most HEP experiments successfully adopted RDBMS technologies (open-source and commercial products) for their needs. At CERN, almost all production systems rely on Oracle databases. For instance, in CMS we use it for dozens of data services, and the two largest databases, DBS and PhEDEx [4], are around a few hundred GBs each excluding indexes.

During Run 2 operations we began seeing limitations of RDBMS-based Run 1 solutions. For instance, in CMS, users are required to place queries across multiple databases to find their desired information. To overcome these obstacles, the CMS experiment has developed a Data Aggregation System [5]. It was designed as an additional layer above existing data services (based on RDBMS backends) and used a NoSQL (MongoDB [6]) database as a caching layer. It aggregates information from different data services, and presents it to end users via the flexible Query Language based on the SQL syntax without explicitly requiring joins between database tables. Even though it was successfully used by CMS in production for many years the growth of information in the experiment requires new solutions to address increasing demand for information, e.g. for monitoring purposes. By the end of Run II, users started becoming more interested in a new type of aggregated information which requires data-processing among distributed databases, joining various attributes, and spanning across large datasets. A typical example would be data popularity plots of user activities for all $\mathrm{T} 1$ and T2 sites over large period of time, e.g. up to a year. To extract this information, it was required to join almost all tables among the three largest databases: the CMS Data Bookkeeping System (DBS), which tracks all experimental datasets, the PhEDEx database, which knows about data location, and the data popularity database which keeps track of user jobs run at various data centers. Such tasks cannot be accomplished via SQL queries since the information physically resides in different databases, and even though Oracle tools provide the ability to perform cross-database joins we found that it does not scale well in practice. Therefore, the process requires manual extraction of relevant information from all databases, proper data-preprocessing, and a complex workflow to obtain desired results. Later, we realized that such a workflow can be easily resolved if all required data will reside on HDFS where we can apply the Spark framework [7] over distributed dataframes and take advantage of parallel processing on an HDFS cluster. Data placement of various metadata sources started in early 2015 and includes several CMS databases, HTCondor logs, CMSSW file access logs, file transfer records as well as Workflow Management logs. At the moment, the CMS experiment has migrated dozens of data sources to HDFS (Table 1) and accumulated more than $32 \mathrm{~TB}$ of data stored in various data formats.

The data on HDFS are stored in various data formats which are suited for different purposes, e.g. $\log$ files are usually streamed to HDFS in native data format (JSON), the database tables are easily converted into CSV data format, while large unstructured data sets, e.g. in 


\begin{tabular}{lll}
\hline \hline HTCondor logs [8] & JSON & $11.1 \mathrm{~TB}$ \\
AAA (Global Data Access) logs [9] & JSON & $11 \mathrm{~TB}$ \\
EOS logs [10] & JSON & $5.3 \mathrm{~TB}$ \\
FTS (File Transfer System) logs [11] & JSON & $4.2 \mathrm{~TB}$ \\
PhEDEx snapshots [4] & CSV & $3.3 \mathrm{~TB}$ \\
WMArchive logs [12] & Avro & $1.3 \mathrm{~TB}$ \\
CMSSW (CMS SoftWare framework) logs & Avro & $0.5 \mathrm{~TB}$ \\
DBS tables [4] & CSV & $0.3 \mathrm{~TB}$ \\
JobMonitoring logs & Avro & $0.2 \mathrm{~TB}$ \\
\hline \hline
\end{tabular}

Table 1. Current snapshot of CMS metadata on HDFS stored on HDFS.

case of the WMArchive system [12], are converted into compact, fast, binary Avro data format with a predefined schema. Fortunately, the HDFS libraries support a broad variety of data formats, and the Spark framework is guaranteed to work seamlessly and efficiently with all of them.

Such availability of large datasets and efficient processing on Hadoop clusters open up new possibilities to push the boundaries of analytics tasks beyond traditional approaches based on relational databases. The run time to spawn TB of data using the Spark framework on HDFS is of the order of a couple of minutes and is not restricted to the content of a single database. Multiple sources can be combined and efficiently processed.

\section{New approaches}

New approaches to handle large datasets in the HEP community are emerging from the business world. First, the NoSQL solutions are adopted to allow storage of unstructured documents, support the distributed natures of databases, and information replication. For example, in CMS we successfully adopted MongoDB [6] and CouchDB [13] technologies for different use cases. The former is used as caching and persistent layers in the Data Aggregation system [5], while the latter is successfully used in Data Management and Workflow Management system [14] to continuously replicate workflows across distributed agents handling CMS Monte Carlo production.

As we mentioned in the previous section the Hadoop ecosystem has begun to play a significant role in almost every HEP experiment. Moreover, the CERN central monitoring system (MONIT) [15] heavily relies on it and incorporates various technologies and tools in their stack, e.g. Kafka, EalsticSearch, InfluxDB, Kibana, Grafana, etc. But all of these innovations come with their own price. Users are required to learn a broad variety of new tools, data formats, etc., and understand how to run their workflows in such environment. While experiments need to adopt their tools and data management systems to new technologies too. For example, in CMS a typical Spark workflow is quite a complex task, see Figure 1. We rely on Python Spark APIs, to read and preprocess data from multiple data-providers on HDFS. The aggregated information is often placed back into the Asynchronous Message Queuing system and ends-up either in an ElasticSearch engine or CERN MONIT systems. Obviously, such a workflow is difficult to construct properly and even harder to maintain on the long run. We often found the majority of tasks are repeated among different users, so a new level of abstraction was desired.

We simplified user access to HDFS, Spark and the CERN Hadoop ecosystem via the CMSSpark framework [16] which takes care of setting up a cluster environment, provides a 


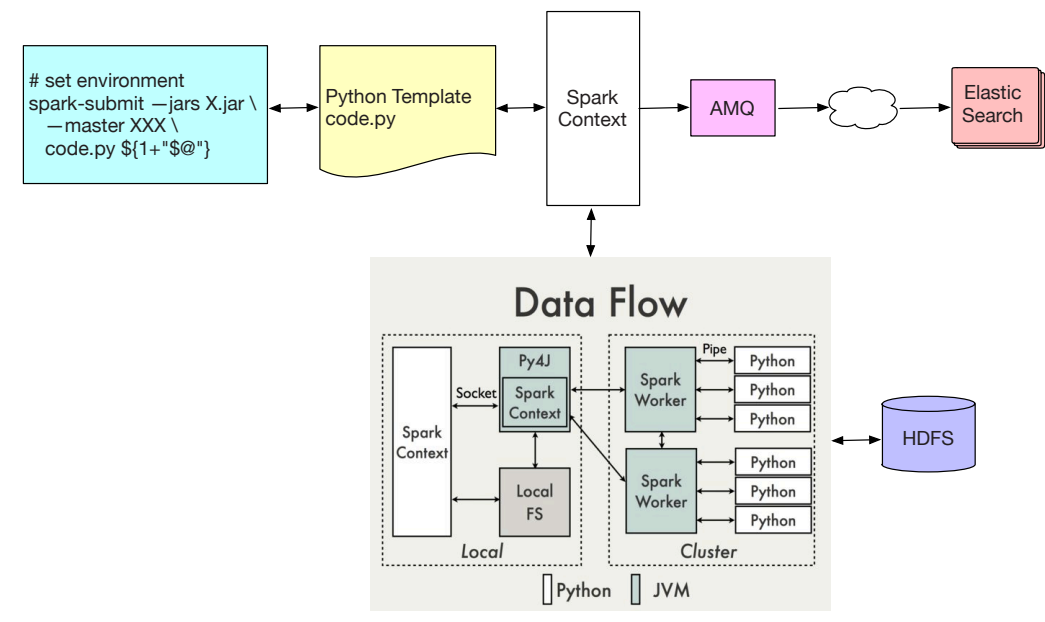

Figure 1. A typical CMSSpark workflow to process data on HDFS cluster via the Spark framework.

layer of abstraction to data access on HDFS, performs data format transformation to Spark DataFrames, handles job handling and data placement back to HDFS and/or CERN MONIT systems. At the end, users are required only to write an analysis code to process the desired DataFrames. A submission of user tasks to the CERN HDFS cluster is simplified to the following command:

\# shell_wrapper + user_workflow + user options run_spark workflow.py --date 20180812 --fout hdfs:///cms/users

Such simplicity has boosted the adaptation of Hadoop tools within the CMS collaboration and was quickly adapted to a variety of use cases, see the discussion in Sect. 3.1.

Although the discussion above was applied to metadata, the Big Data tools can also help the HEP community apply new techniques to process and analyze real data. For instance, the authors of [2] proposed to use HDFS/Hadoop as a data-reduction facility in HEP analysis with the ambitious goal of reducing $1 \mathrm{~PB}$ of raw input data to 1TB output data in a few hours. This approach can be further extended not only to HEP analysis per-se, but also to train Machine Learning (ML) models on petabyte datasets.

The ML models in the HEP community have been used for years, e.g. Boosted Decision Trees or simple Neural Networks which are often used in various physics analysis. The recent advances of technologies both on hardware and software fronts allow ML models to be adopted universally, from computing infrastructure to trigger systems [17]. But the key problem with ML training is the data preparation step which involves data transformation and preprocessing. The most common data format for ML training is CSV (or alike) while the HEP data are stored in ROOT data format. Recent developments in ROOT I/O [18] and ROOT data access [19] open up a possibility to directly read and process ROOT data on the HDFS cluster. With this change we are already able to organize new types of workflows of reading petabytes of data, perform necessary data transformation and preprocessing on HDFS, train ML models, and deliver them to end users as a data service [20]. Figure 2 demonstrates such an R\&D pipeline in the context of the TFaaS project for the CMS experiment.

Preliminary studies shows that we can achieve reading HEP events at the rate of $100 \mathrm{kHz}$ $(50 \mathrm{MB} / \mathrm{s})$ via the uproot [21] library, preprocess TB of data in a range of minutes to a few 


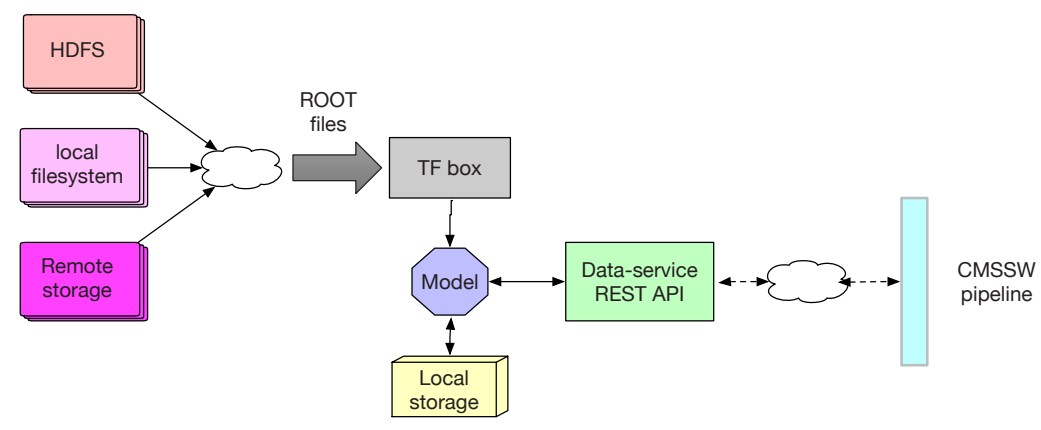

Figure 2. A TensorFlow as a Service [20] architecture for a HEP use case. The input raw data in ROOT data format can be read from remote data-providers, including XRootD servers, local filesystems, and HDFS, be processed and transformed on the HDFS/Spark platform and fed into ML framework for training (e.g. via SparkML). The trained model can be served to end users or the entire framework (CMSSW) as an HTTP based data service.

minutes to few hours ${ }^{2}$ on the HDFS cluster [22], train a model and serve it to end users via TensorFlow as a Service [20] tool with high throughput in a distributed environment. Our benchmarks have shown that we can easily achieve $500 \mathrm{req} / \mathrm{s}$ throughput for concurrent clients using a single node serving TFaaS data service.

These new approaches are developed rapidly and are partially adopted in the CMS experiment. Below we briefly discuss a few of them in the context of the CMS monitoring infrastructure.

\subsection{CMS monitoring}

The CMS monitoring infrastructure is gradually migrating from experiment specific tools to the central CERN Monitoring system [15]. The CERN MONIT system consists of more than a hundred data producers with 3.5 TB/day injection rate and dozens of Spark jobs running 24/7. It is highly integrated with the CERN Analytix cluster composed of 39 nodes with 64GB of RAM, 32 cores/node Mix of Intel® Xeon® CPU E5-2650 @ 2GHz AMD Opteron $\left({ }^{\mathrm{TM}}\right) 6276$, and capable of storing and handling PB of data.

So far we have migrated several experiment dashboards to the CERN MONIT infrastructure, among them AAA, EOS, HTCondor, task monitoring of user analysis jobs as well as WMArchive subsystems. We found that the Spark platform significantly improved our analytics capabilities. For instance, in the WMArchive [12] system we can promptly perform the following tasks:

- identify failed workflows and problematic sites

- spot production issues via log look-up and exit codes

- monitor CMS production status, including sites, campaigns monitoring and extracting throughput metrics

- perform data aggregation and produce aggregated statistics.

The system was designed to collect $100 \mathrm{M}+$ documents per year from distributed WMAgents with an upload rate of $O(1 M)$ documents per day. The documents were injected into the local cache of MongoDB and are transferred to HDFS for long-term storage. We periodically run

\footnotetext{
${ }^{2}$ The processing time strongly depends on the specific use case and complexity of the executed workflow.
} 
daily and hourly aggregation jobs to gain insight on Monte Carlo production workflows. This information is fed into the CERN MONIT system where it is displayed in various dashboards.

Using CMS data on HDFS, as outlined Table 1, we analyzed the most popular data tier among end users in 2017. To our satisfaction it was MINIAODSIM accessed by $56 \%, 42 \%$, $40 \%, 40 \%$ in AAA, EOS, CMSSW, and CRAB systems, respectively. Then it was followed by MINIAOD, AOD, and RAW data-tiers. The usage of the RECO data-tier was quite negligible, at the level of a few percent in corresponding systems.

We also look at the data popularity content of our data and successfully used the Hadoop Spark platform as a data reduction and processing facility. We performed studies to predict dataset popularity using user AAA logs [22]. We demonstrated that it can be modeled via ML and be used as a seed by the CMS dynamic data placement system. It worth mentioning that the original dataset had $2 \mathrm{~B}$ rows of AAA logs combined with PhEDEx database tables. This dataset was reduced to $0.5 \mathrm{M}$ records in a couple of hours and fed into the SparkML framework for training the ML model. We foresee that such a workflow pipeline can be successfully adopted in the HL-LHC era where intelligent data placement may play a critical role.

Finally, we used Job Monitoring and WMArchive data to measure sites performance. The studies targeted a concrete architecture on T2 sites where node throughput was calculated as a number of processed events per second for various processor architectures taking into account the number of job slots per core. These results complemented well established HS06 scores and were included in the HEPiX Benchmarking Working Group [23].

\section{Summary}

The CMS experiment is continuously improving its computing and offline infrastructure. In particular, it is shifting its monitoring infrastructure to the central CERN monitoring system and has a large number of ML projects.

In this paper we discussed a gradual shift to handle large datasets in the CMS experiment. The large portion of CMS metadata has been successfully migrated to HDFS and complements our existing database solutions. The usage of Hadoop tools and its ecosystem is no longer a problem due to the simplicity of the CMSSpark framework. It simplified access to a broad variety of CMS data located on HDFS by abstracting the data access layer, and provided a simple and uniform way to submit, process, and analyze these data.

We also described a new use case for Machine Learning training over large distributed datasets and are continuously delivering ML models to end users via a new Tensor as a Service data platform recently developed and which is currently undergoing testing in the CMS experiment. Such a service may not only serve the CMS experiment per-se but can be applicable to other experiments. It may fill the gap of integrating ML tools into the existing infrastructure and experiment framework. Finally, we foresee that this approach will gain popularity in the upcoming years of data taking in the HL-LHC regime where training ML models over petabyte datasets will become a norm.

\section{Acknowledgement}

I would like to thank my CMS colleagues David Lagne (Princeton) and Carl Vuosalo (Univ. of Wisconsin) for their support and various contributions in CMS monitoring infrastructure, including production and validation of of T1/T2 usage plots. I also would like to thank Luca Menichetti from CERN IT who provided support for development, maintenance and deployment of our scripts on the Spark platform. Special thanks go to the CERN MONIT team for collaboration and support to set up and maintain the CMS Monitoring dashboards. 


\section{References}

[1] A. A. Alves Jr., et. al., A Roadmap for HEP Software and Computing RED for the 2020s, https://arxiv.org/abs/1712.06982

[2] O. Gutche, et al., Big Data in HEP: A comprehensive use case study, https://arxiv.org/abs/1703.04171

[3] E.F. Codd, A Relational Model of Data for Large Shared Data Banks, Communications of the ACM. 13 (6): 377-387. doi:10.1145/362384.362685.

[4] M. Giffels, Y. Guo, V. Kuznetsov, N. Magini and T. Wildish The CMS Data Management System J. Phys.: Conf. Ser. 513 (2014) 042052; doi:10.1088/1742-6596/513/4/042052

[5] V. Kuznetsov, D. Evans, S. Metson, The CMS Data Aggregation System, doi:10.1016/j.procs.2010.04.172

[6] MongoDB document-oriented database, https://docs.mongodb.org, accessed (2019)

[7] Apache Spark, https://spark.apache.org, accessed (2019)

[8] D. Thain, T. Tannenbaum and M. Livny, Distributed computing in practice: the Condor experience Concurrency and Computation: Practice and Experience, (2005) 17 2-4 323356 doi:10.1002/cpe.938

[9] K. Bloom, et. al., Any Data, Any Time, Anywhere: Global Data Access for Science, BDC (2015), 85-91 https://arxiv.org/pdf/1508.01443.pdf

[10] X. Espinal, et. al., Disk storage at CERN: Handling LHC data and beyond Journal of Physics: Conference Series 513 (2014) 042017 doi:10.1088/1742-6596/513/4/042017

[11] A A Ayllon, et. al., FTS3: New Data Movement Service For WLCG, J. Phys. Conf. Ser. 513 (2014) 032081

[12] V. Kuznetsov, N. Fischer, Y. Guo, The archive solution for distributed workflow management agents of the CMS experiment at LHC Computing and Software for Big Science (2018), 2:1, doi: 10.1007/s41781-018-0005-0

[13] Apache CouchDB data-management system, http://couchdb.apache.org, accessed (2019)

[14] M. Cinquilli, et. al., The CMS workload management system, Journal of Physics: Conference Series (2012), Volume 396, Part 3

[15] A. Aimar, et. al., Unified Monitoring Architecture for IT and Grid Services Journal of Physics Conference Series (2017), 898 092033, doi: 10.1088/1742-6596/898/9/092033

[16] V. Kuznetsov, CMSSpark a general purpose framework to run CMS experiment workflows on HDFS/Spark platform DOI 10.5281/zenodo.1401228 https://zenodo.org/badge/latestdoi/74044584

[17] K. Albertsson, et. al., Machine Learning in High Energy Physics Community White Paper https://arxiv.org/abs/1807.02876

[18] B. Bockelman, Z. Zhang, J. Pivarski, Optimizing ROOT IO For Analysis, https://arxiv.org/abs/1711.02659

[19] J. Pivarski, P. Elmer, B. Bockelman, Z. Zhang, Fast Access to Columnar, Hierarchical Data via Code Transformation https://arxiv.org/pdf/1708.08319.pdf

[20] V. Kuznetsov, TensorFlow as a Service doi: 10.5281/zenodo.1308048

[21] DIANA-HEP Scikit-hep uproot library, Minimalist ROOT I/O in pure Python and Numpy, https://github.com/scikit-hep/uproot, accessed (2019)

[22] M. Meoni, V. Kuznetsov, L. Menichetti, J. Rumševičius, T. Boccali, D. Bonacorsi, Exploiting Apache Spark platform for CMS computing analytics, ACAT (2017), http://arxiv.org/abs/1711.00552 
[23] HEPiX Benchmark and Performance group, https://w3.hepix.org/benchmarking.html, accessed (2019) 